\title{
On the theory of autosoliton propagation in optical fibers guided by in-line nonlinear devices
}

\author{
Sonia Boscolo, ${ }^{a}$ Stanislav A. Derevyanko, ${ }^{a}$ Sergei K. Turitsyn, ${ }^{a}$ \\ Alexander S. Kovalev, ${ }^{b}$ and Mikhail M. Bogdan ${ }^{b}$ \\ ${ }^{a}$ Photonics Research Group, School of Engineering and Applied Science, \\ Aston University, Birmingham B4 7ET, United Kingdom; \\ ${ }^{b}$ B. I. Verkin Institute for Low Temperature Physics and Technology, \\ 47 Lenin Ave, 61103 Kharkov, Ukraine
}

\begin{abstract}
A theoretical model is developed to describe the propagation of ultra-short optical pulses in fiber transmission systems in the quasi-linear regime, with periodically inserted in-line lumped nonlinear optical devices. Stable autosoliton solutions are obtained for a particular application of the general theory.
\end{abstract}

Keywords: Autosolitons, fiber-optics communications, nonlinear optical devices, quasi-linear propagation regime

\section{INTRODUCTION}

Efficient growth of the capacity of digital communication systems can be achieved by increase of the channel bit rate - the speed at which information symbols are transmitted. Current commercial optical fiber systems, responsible for the major part of the total world data traffic, operate at channel rates up to $10 \mathrm{Gbit} / \mathrm{s}$, while $40 \mathrm{Gbit} / \mathrm{s}$ lines are already ready for installation and $160 \mathrm{Gbit} / \mathrm{s}$ links are a subject of intensive laboratory research. Increasing the channel rate assumes the utilization of shorter time slots allocated for each information bit and, consequently, of shorter carrier pulses. The propagation of ultra-short pulses is strongly affected by the fiber dispersion, which results in large temporal broadening of the carrier pulses. The accumulated line dispersion is typically compensated periodically or at the edges of the link, thus, in the main linear approximation, the pulse width is almost recovered at the end of the transmission line. Because of the temporal broadening during propagation, the carrier pulse power spreads over many time slots and, consequently, the accumulated effect of the instantaneous fiber nonlinearity tends to get averaged out. Signal transmission using very short optical pulses is often referred to as the quasi-linear regime. ${ }^{1}$ This regime is, in some sense, opposite to soliton ${ }^{2}$ or dispersion-managed (DM) soliton ${ }^{3}$ transmission, where fiber nonlinearity plays an important role in preserving the pulse shapes during propagation. Note that in the quasi-linear regime, the in-line Kerr nonlinearity is almost a "negative" factor contributing to the destabilization and distortion of carrier pulses. Therefore, a certain amount of "constructive" nonlinearity is required to stabilize ultra-short pulse propagation and, thus, to improve the system performance. Recently, the periodic in-line deployment of nonlinear optical devices (NODs), such as nonlinear optical loop mirrors (NOLMs), semiconductor saturable absorbers, and semiconductor amplifierbased devices, has been demonstrated to be an effective technique of all-optical signal regeneration, ${ }^{4-6}$ which may achieve stable pulse propagation and virtually unlimited transmission distances in high-speed, strongly DM optical fiber communication systems. ${ }^{5}$ It has numerically been shown in Refs. 4,5 that, under certain conditions, the interplay between fiber dispersion, the lumped nonlinearity provided by in-line NOLMs, and the action of linear control elements, such as optical filters, leads to the formation of autosolitons, which are periodically reproduced at the output of each segment of the transmission line. The term 'autosolitons' here means robust localized pulses with the parameters prescribed by properties of the system, which occur in models combining conservative and dissipative dispersive and nonlinear terms. ${ }^{7-9}$

S. Boscolo: E-mail: boscolsa@aston.ac.uk

S. A. Derevyanko: E-mail: s.derevyanko@aston.ac.uk

S. K. Turitsyn: E-mail: s.k.turitsyn@aston.ac.uk

ICONO 2005: Nonlinear Space-Time Dynamics, edited by Nikolai Rosanov, Stephano Trillo, Proc. of SPIE Vol. 6255, 625502, (2006) · 0277-786X/06/\$15 - doi: 10.1117/12.676464 
The use of ultra-short optical pulses in fiber-optics communication leads to new interesting physical regimes and novel mathematical models should be introduced to adequately describe such transmission systems. Note that further increase of the channel bit rate will lead to even shorter carrier pulses and more pronounced quasilinear propagation regimes in the fiber parts of communication systems. In this paper, a theory is developed to describe the optical signal transmission in DM fiber transmission systems in the quasi-linear regime, with periodically in-line placed point NODs. We present a fundamental discrete mapping equation governing the carrier pulse propagation in a unit cell of the transmission line. As a particular sample approach to the solution of this basic model, we apply a variational method to determine the steady state pulse characteristics. Without loss of generality, as a specific practical application of the general theory, we consider a system with in-line NOLMs.

\section{THEORETICAL MODEL}

The optical pulse propagation in a cascaded transmission system with periodic variations of dispersion and nonlinearity, frequency filtering, and NOD management can be described by

$$
i \frac{\partial E}{\partial z}-\frac{1}{2} \beta_{2}(z) \frac{\partial^{2} E}{\partial t^{2}}+\sigma(z)|E|^{2} E=i G\left(z,|E|^{2}\right) E,
$$

where $E(z, t)$ is the slowly varying pulse envelope in the comoving system of coordinates, $\beta_{2}(z)$ represents the variation in the group-velocity dispersion due to dispersion compensation, and is assumed to be a periodic function of $z$ with the period $L, \beta_{2}(z)=\beta_{2}(z+L)$, and $\sigma$ is the fiber nonlinear coefficient. It is customary to express $\beta_{2}$ in terms of the associated dispersion coefficient $D$ via $\beta_{2}=-\lambda^{2} D /\left(2 \pi c_{0}\right)$, where $\lambda$ is the carrier wavelength, $c_{0}$ is the speed of light, and $D$ is measured in $\mathrm{ps} /(\mathrm{nm} \mathrm{km})$. Function $G\left(z,|E|^{2}\right)$ accounts for the signal attenuation due to fiber loss, the signal amplification by optical amplifiers, the action of filters, and the nonlinear gain at the NODs, and can be presented as

$$
\begin{aligned}
G\left(z,|E|^{2}\right)= & -\gamma(z)+\sum_{k} \delta\left(z-k Z_{a}\right)\left\{\exp \left[\int_{(k-1) Z_{a}}^{k Z a} \mathrm{~d} z \gamma(z)\right]-1\right\} \\
& +\sum_{k} \delta\left(z-k Z_{f}\right)[h(t) *-1]+\sum_{k} \delta\left(z-k Z_{0}\right)\left[f\left(|E|^{2}\right)-1\right] .
\end{aligned}
$$

In Eq. (2), we have assumed that amplifiers, filters, and NODs are placed periodically in the system with the respective periods $Z_{a}, Z_{f}$, and $Z_{0} \cdot \gamma=0.05 \ln (10) \alpha$ is the fiber loss coefficient that accounts for the signal attenuation along the fiber span before the $k$ th amplifier, $\alpha$ is given in $\mathrm{dB} / \mathrm{km}$, and $\exp \left[\int_{(k-1) Z_{a}}^{k Z_{a}} \mathrm{~d} z \gamma(z)\right]-1$ is the amplification coefficient after the fiber span between the $(k-1)$ th and $k$ th amplifiers. $h(t)$ is the inverse Fourier transform of the filter transfer function, and $*$ represents the Fourier convolution. The NODs are specified by their power-dependent transfer function $f(P)$. Hereafter, we will focus on loss (gain)-unbalanced fiber NOLMs. The transfer function for such devices can be written in the form

$$
f(P)=a \sin (b P) \exp (i c P),
$$

with $a, b, c \in \Re^{+}$some given constants.

To simplify the full model given by system (1), we make some justified physical assumptions. Here, we analyze the case of linear propagation in fiber, when we can neglect the nonlinear term in (1). Such a propagation regime corresponds to the case when the nonlinear length $L_{\mathrm{NL}}=\left(\sigma P_{0}\right)^{-1}\left(P_{0}\right.$ is the signal peak power) in the fiber is much larger than the local dispersion length $L_{\mathrm{D}}=T^{2} /\left|\beta_{2}\right|$ (T is the pulse width). The transformation of a pulse after propagation in one segment of the transmission line can be considered as the mapping of the input pulse into the output one. If we consider an element of the transmission line that includes a NOD given by (3), a piece of linear fiber of length $Z_{0}$, and $m$ filters, the mapping of the signal, defined up to a phase factor $\mu$, can be presented as

$$
e^{i \mu} U_{n+1}(t)=\int_{-\infty}^{+\infty} \mathrm{d} t^{\prime} K\left(t-t^{\prime} ; Z_{0}\right) f\left(\left|U_{n}\left(t^{\prime}\right)\right|^{2}\right) U_{n}\left(t^{\prime}\right), \quad n=0,1, \ldots
$$


The derived equation is one of the central results of the paper. This mapping problem plays a fundamental role in the description of fiber communication systems at high bit rates. To obtain Eq. (4), we have assumed that each NOD is placed immediately after an amplifier, and we have applied the transformation $U(z, t)=Q^{-1}(z) E(z, t)$, where $Q(z)=\exp \left[-\int_{(k-1) Z_{a}}^{z} \mathrm{~d} z^{\prime} \gamma\left(z^{\prime}\right)\right]$ for $(k-1) Z_{a}<z<k Z_{a}$, and $Q(z)=1$ for $z=k Z_{a}^{+}$. In Eq. (4), the signal is taken at the input point $n Z_{0}^{-}$to the NOD after any device prior to the NOD, and the kernel $K$ describes the signal propagation in the unit cell $Z_{0}$,

$$
K\left(t-t^{\prime} ; Z_{0}\right)=\frac{1}{2 \pi} \int_{-\infty}^{+\infty} \mathrm{d} t^{\prime \prime} H\left(t-t^{\prime}-t^{\prime \prime}\right) F\left(t^{\prime \prime} ; Z_{0}\right)
$$

where $H(t)$ represents the action of the filters,

$$
H(t)=\mathcal{F}^{-1}\left[\hat{h}^{m}(\omega)\right]
$$

and $F\left(t ; Z_{0}\right)$ is the linear propagator of the uniform equation corresponding to Eq. (1),

$$
F\left(t ; Z_{0}\right)=\sqrt{\frac{i}{B_{0}}} \exp \left(-\frac{i t^{2}}{2 B_{0}}\right), \quad B_{0}=\int_{n Z_{0}}^{(n+1) Z_{0}} \mathrm{~d} z \beta_{2}(z) .
$$

In (7), $B_{0}$ is the total accumulated dispersion. Note that $H(t) \rightarrow \sqrt{2 \pi} \delta(t)$ when $m \rightarrow 0$ (no filtering), and $F\left(t ; Z_{0}\right) \rightarrow \sqrt{2 \pi} \delta(t)$ when $B_{0} \rightarrow 0$ (full dispersion compensation). In the case when Gaussian filters are used, it is easy to verify that $K$ takes the form

$$
K\left(t-t^{\prime} ; Z_{0}\right)=\sqrt{G} \sqrt{\frac{i}{2 \pi\left(B_{0}+i m / \Omega_{f}^{2}\right)}} \exp \left[-\frac{i\left(t-t^{\prime}\right)^{2}}{2\left(B_{0}+i m / \Omega_{f}^{2}\right)}\right],
$$

where $\Omega_{f}=\pi \delta \nu_{f} / \sqrt{\ln 2}$ is the filter bandwidth ( $\delta \nu_{f}$ denotes the full-width at half-maximum (FWHM) bandwidth), and the excess gain $G$ accounts for compensation of the signal energy losses introduced by the NODs and filters in the system.

From the transmission point of view it is desirable to find (if exists) a steady state propagation regime in which an optical pulse propagating along the transmission line reproduces periodically at the output of each element of the line. That corresponds to determining a fixed point of mapping (4). Therefore, in order to find the steady state pulse shape $U(t)$, one has to solve a nonlinear integral equation, which stems from (4) if we put $U_{n+1}(t)=U_{n}(t)=U(t)$. If the steady state pulse is stable, then any initial signal within the basin of attraction of the fixed point will gradually evolve towards it after some maps.

\section{AUtOSOLitons}

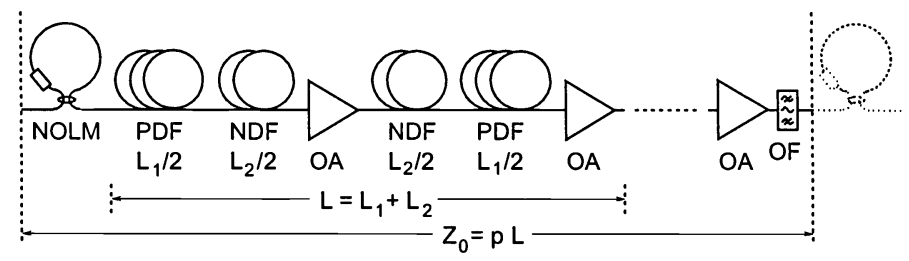

Figure 1. One element of the periodic transmission system.

In this section, we demonstrate the feasibility of stable autosoliton propagation guided by in-line NOLMs, by direct numerical simulations of the basic propagation model (Eq. (1)). The sample transmission scheme used in the numerical integration of Eq. (1) is depicted in Fig. 1. The transmission line is composed of an 
equal number of positive (anomalous) dispersion fiber (PDF) segments and negative (normal) dispersion fiber (NDF) segments. The dispersion map consists of an alternation of a PDF-NDF block and a mirror NDF-PDF block. Fiber parameters of practical importance $\left(D_{1}=15 \mathrm{ps} /(\mathrm{nm} \mathrm{km}), \sigma_{1}=1.84(\mathrm{~W} \mathrm{~km})^{-1}, \alpha_{1}=0.22 \mathrm{~dB} / \mathrm{km}\right.$; $\left.D_{2}=-71.2 \mathrm{ps} /(\mathrm{nm} \mathrm{km}), \sigma_{2}=4.28(\mathrm{~W} \mathrm{~km})^{-1}, \alpha_{2}=0.65 \mathrm{~dB} / \mathrm{km}\right)$ are used for the PDF and the NDF. ${ }^{5}$ We note that fiber nonlinearity is included in the calculations. An optical amplifier (OA), which compensates for the fiber loss, follows each of the two blocks. The high values of the local dispersion of the fibers together with the short pulse widths that are typically used to operate the system at high data transmission rates result in large broadening of the pulses during propagation. These regimes are beyond the range where stable propagation of DM solitons has been observed. ${ }^{10}$ A NOLM is placed into the transmission line every an integer number $p$ of dispersion map periods, $Z_{0}=p L$. We note that in this case $B_{0}=\left\langle\beta_{2}\right\rangle Z_{0}=-\lambda^{2}\langle D\rangle Z_{0} /\left(2 \pi c_{0}\right)(\langle\cdot\rangle$ denotes the average over the dispersion compensating period $L)$. In the sample configuration considered here, $Z_{0}=391 \mathrm{~km}$, and $p=5$. A single $(m=1)$ Gaussian optical filter (OF) is placed after the amplifier prior to the NOLM location. The loss-unbalanced NOLM configuration is employed as an example, and preamplification of the input pulses to the NOLM is used (see Ref. 5 for details). Parameters $a, b$, and $c$ in transfer function (3) have the respective values: $0.06373,1.823 \mathrm{~W}^{-1}$, and $1.839 \mathrm{~W}^{-1}$. The NOLM acts as a saturable absorber and, hence, filters out lowintensity radiation from the higher-power pulses. This allows for restoration of the pulse amplitude and shape. ${ }^{4,5}$ Following Ref. 5, the system is operated such that the peak power of the steady state pulses (if any exist) is in the region slightly past the first peak of the continuous-wave power characteristic of the NOLM. In this region the NOLM provides a negative feedback control of the amplitude of pulses, which may enable stabilization of the pulse amplitude fluctuations. ${ }^{5}$ Of course, such a consideration does not guarantee stable pulse propagation in the system, and is used here only to reduce the space of system parameters where the steady state pulses are searched for.
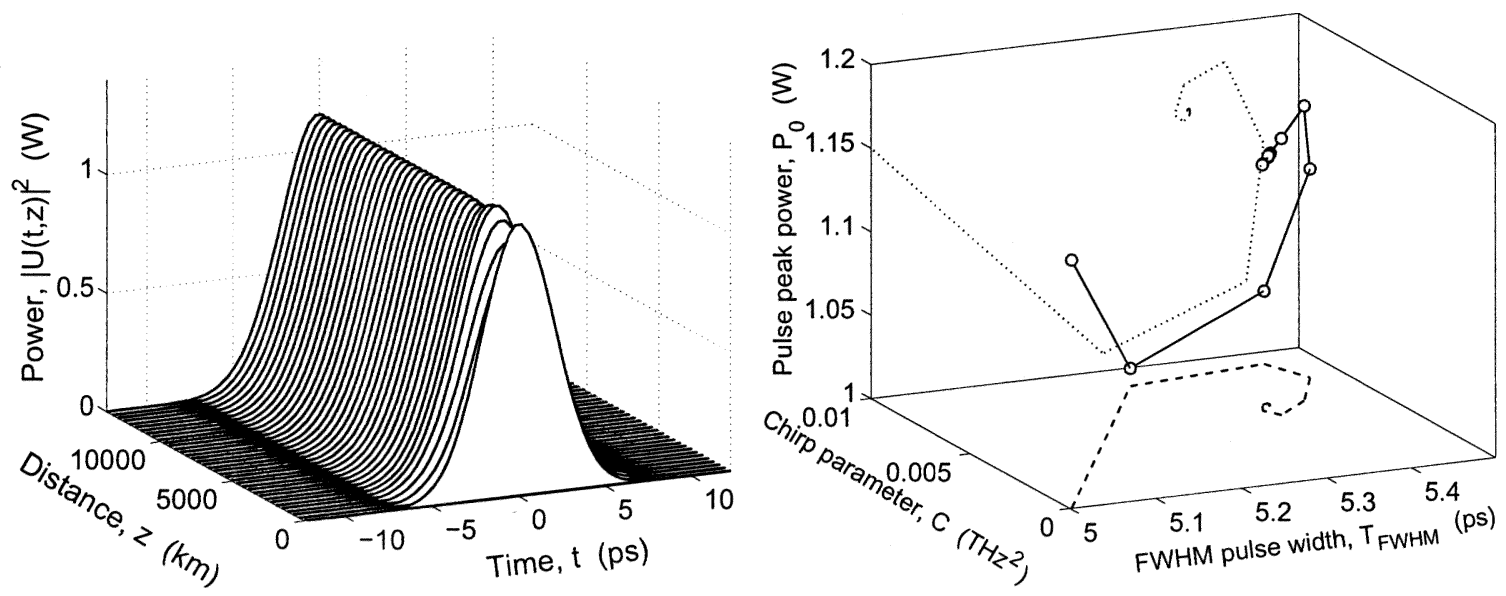

Figure 2. Stroboscopic pulse evolution as viewed at the NOLM input. Left, evolution of the intensity profile; right, acqusition of the steady state in the space of pulse parameters.

Figure 2 shows an example of pulse evolution in the system, measured stroboscopically at the NOLM input point. In this example, an unchirped Gaussian pulse is launched into the system, with the peak power $P_{0}=$ $1.15 \mathrm{~W}$ (corresponding to $3.5 \mathrm{~mW}$ at the starting point of the transmission) and the FWHM pulse width $T_{\mathrm{FWHM}}=$ 5 ps. The system parameters are: $\langle D\rangle=0.009 \mathrm{ps} /(\mathrm{nm} \mathrm{km}), \delta \nu_{f}=\sqrt{\ln 2} \Omega_{f} / \pi=0.1 \mathrm{THz}$, and $G=627.0$ $(28.0 \mathrm{~dB})$. The pulse chirp parameter is calculated as $C=\operatorname{Im} \int_{-\infty}^{+\infty} \mathrm{d} t U^{2}\left(U_{t}^{*}\right)^{2} / \int_{-\infty}^{+\infty} \mathrm{d} t|U|^{4}$. One may see from Fig. 2 that the pulse settles to a steady state after a short initial transition distance. This result demonstrates the feasibility of stable pulse propagation in the system, and indicates that the use of in-line NOLMs converts the quasi-linear transmission regime into an autosoliton transmission regime, which is strictly nonlinear. ${ }^{5}$ We note that the same stroboscopic picture as that in Fig. 2 can be obtained by simply iterating mapping equation (4). Figure 3 shows the basin of attraction of the steady state solution of Fig. 2 in the plane $\left(T_{\mathrm{FWHM}}, C\right)$. To 


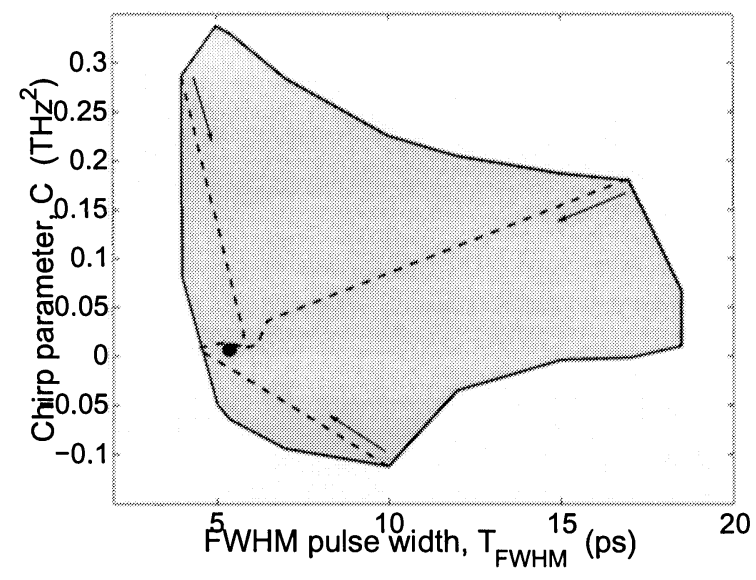

Figure 3. Basin of attraction of the steady state solution of Fig. 2 in the plane FWHM pulse width-chirp parameter.

calculate Fig. 3, the initial pulse peak power has been set to its steady state value. It is seen that there is a large tolerance to the initial pulse width and chirp, which indicates a high degree of stability of the steady state solution. We have also defined the tolerable limits of the stable pulse propagation to the filter bandwidth and the path-averaged dispersion of the line. The results are shown in Fig. 4, where $\delta \nu_{f}$ and $\langle D\rangle$ are varied within a practical range of values. In Fig. 4 , the excess gain $G$ is chosen such that the stationary pulse peak power is approximately $1.15 \mathrm{~W}$ at the NOLM input.

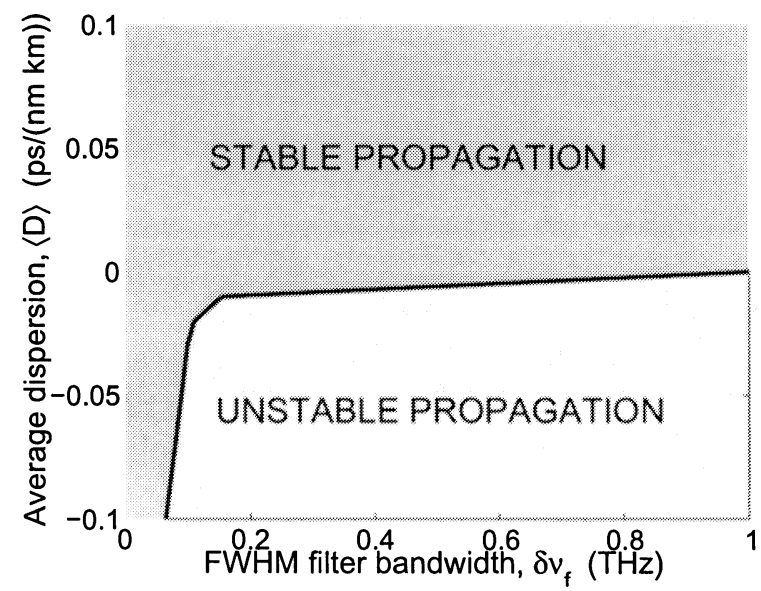

Figure 4. Limits of stable pulse propagation in the plane filter bandwidth-average dispersion.

\section{VARIATIONAL APPROACH}

As we pointed out previously, in order to find a steady state pulse propagation regime, one has to solve mapping integral equation (4) for the fixed points. While this can be done numerically, for massive optimization of the system parameters it is very useful to have a simplified approximate method to find solutions of the basic model. Therefore, here we apply a simple variational approach. We noticed from full numerics that the steady state pulse shape at the NOLM input point can always be fitted well by a Gaussian profile. Thus, we choose a trial 
input pulse $U(t)$ for the map as a Gaussian-shaped pulse with (yet unknown) peak power $P_{0}$, root-mean-square (RMS) width $T_{\mathrm{RMS}}$, and RMS chirp parameter $C_{\mathrm{RMS}}$,

$$
U_{n}(t)=\sqrt{P_{0}} \exp \left(-\frac{t^{2}}{4 T_{\mathrm{RMS}}^{2}}+i C_{\mathrm{RMS}} t^{2}\right) .
$$

The output of the map $U_{n+1}(t)$ given by Eq. (4) will be non-Gaussian in general, but will have a close shape and will depend on the parameters of the input signal, $U_{n+1}=U_{n+1}\left(t ; P_{0}, T_{\mathrm{RMS}}, C_{\mathrm{RMS}}\right)$. Let us now demand that the peak power, pulse width, and chirp of the output signal coincide with those of the input Gaussian signal. This provides a system of transcendental equations for the sought parameters $P_{0}, T_{\mathrm{RMS}}$, and $C_{\mathrm{RMS}}$,

$$
\begin{gathered}
P_{0}=\frac{1}{\sqrt{2 \pi} T_{\mathrm{RMS}}} \int_{-\infty}^{+\infty} \mathrm{d} t\left|U_{n+1}\left(t ; P_{0}, T_{\mathrm{RMS}}, C_{\mathrm{RMS}}\right)\right|^{2}, \quad T_{\mathrm{RMS}}^{2}=\frac{\int_{-\infty}^{+\infty} \mathrm{d} t t^{2}\left|U_{n+1}\left(t ; P_{0}, T_{\mathrm{RMS}}, C_{\mathrm{RMS}}\right)\right|^{2}}{\int_{-\infty}^{+\infty} \mathrm{d} t\left|U_{n+1}\left(t ; P_{0}, T_{\mathrm{RMS}}, C_{\mathrm{RMS}}\right)\right|^{2}}, \\
C_{\mathrm{RMS}}=\frac{\operatorname{Im} \int_{-\infty}^{+\infty} \mathrm{d} t U_{n+1}^{2}\left(t ; P_{0}, T_{\mathrm{RMS}}, C_{\mathrm{RMS}}\right)\left(\partial_{t} U_{n+1}^{*}\left(t ; P_{0}, T_{\mathrm{RMS}}, C_{\mathrm{RMS}}\right)\right)^{2}}{\int_{-\infty}^{+\infty} \mathrm{d} t\left|U_{n+1}\left(t ; P_{0}, T_{\mathrm{RMS}}, C_{\mathrm{RMS}}\right)\right|^{4}} .
\end{gathered}
$$

If the solution of system (10) exists, then it provides a variational approximation for the parameters of the steady state pulse. In particular, one may use the Gaussian ansatz with the found vaues of $P_{0}, T_{\mathrm{RMS}}$, and $C_{\mathrm{RMS}}$ as an approximation of the steady state pulse shape.


Figure 5. Steady state pulse peak power (left), RMS pulse width and RMS chirp parameter (right) at the NOLM input versus filter bandwidth.

The predictions form the variational model (Eqs. (9), (10)) and the iterative numerical integration of mapping equation (4) have been compared with the results of full numerical simulations. The steady state pulse peak power, RMS width, and RMS chirp parameter are plotted in Fig. 5 as a function of the filter bandwidth, for $\langle D\rangle=0.009 \mathrm{ps} /(\mathrm{nm} \mathrm{km})$, and the same values of $G$ as used in Fig. 4 . The results agree well, especially for the pulse width and the chirp parameter. As for the pulse amplitude, the discrepancies between the solutions of Eq. (4) and full numerics occurring for small values of $\delta \nu_{f}$ (of the order of $6 \%$ at maximum) can be attributed to the fact that for small filter bandwidths the effect of the nonlinearity in the transmission fibers, which is neglected in model (4), becomes more important. For larger $\delta \nu_{f}$, the deviation of model (4) from full numerics does not exceed $0.6 \%$, which fully justifies the linear assumption underlying Eq. (4) in this filter bandwidth range. The deviation of the variational model from either full numerics or model (4) does not exceed $2 \%$ in the bandwidth range where the linear assumption is entirely justified. 


\section{CONCLUSION}

We have developed a theoretical model to describe the ultra-short pulse propagation in fiber transmission systems in the quasi-linear regime, with periodic in-line deployment of NODs. In the particular application with NOLMs, we have numerically demonstrated that formation of autosolitons can be observed in such systems, as a result of a balance between the effects of dispersion in the transmission fibers, linear control by optical filters, and nonlinear focusing in the NOLMs. A variational principle has been applied to determine the steady state pulse characteristics, and the theoretical analysis has been shown to accurately reproduce the results of full numerical simulations.

\section{REFERENCES}

1. P. V. Mamyshev and N. A. Mamysheva, "Pulse-overlapped dispersion-managed data transmission and intrachannel four-wave mixing," Opt. Lett. 24, pp. 1454-1456, 1999.

2. A. Hasegawa and Y. Kodama, Solitons in Optical Communications, Oxford University Press, Oxford, 1995.

3. I. Gabitov and S. K. Turitsyn, "Averaged pulse dynamics in a cascaded transmission system with passive dispersion compensation," Opt. Lett. 21, pp. 327-329, 1996.

4. S. Boscolo, J. H. B. Nijhof, and S. K. Turitsyn, "Autosoliton transmission in dispersion-managed systems guided by in-line nonlinear optical loop mirrors," Opt. Lett. 25, pp. 1240-1242, 2000.

5. S. Boscolo, S. K. Turitsyn, and K. J. Blow, "Study of the operating regime for all-optical passive 2R regeneration of dispersion-managed RZ data at $40 \mathrm{~Gb} / \mathrm{s}$ using in-line NOLMs," IEEE Photon. Technol. Lett. 14, pp. 30-32, 2002.

6. D. Rouvillain, P. Brindel, E. Seguineau, L. Pierre, O. Leclerc, H. Choumane, G. Aubin, and J. L. Oudar, "Optical 2R regenerator based on passive saturable absorber for $40 \mathrm{Gbit} / \mathrm{s}$ WDM long-haul transmissions," Electron. Lett. 38, pp. 1113-1114, 2002.

7. B. S. Kerner and V. V. Osipov, Autosolitons, Kluwer Academic Publ., Dordrecht, 1994.

8. V. S. Grigoryan, "Asymptotically stable solitary waves in a Kerr dispersive medium in the presence of linear amplification and nonlinear losses," Phys. Rev. A 52, pp. 694-698, 1995.

9. A. G. Vladimirov, G. V. Khodova, and N. N. Rosanov, "Stable bound states of one-dimensional autosolitons in a bistable laser," Phys. Rev. E 63, 056607, 2001.

10. J. H. B. Nijhof, N. J. Doran, W. Forysiak, and A. Berntson, "Energy enhancement of dispersion-managed solitons and WDM," Electron. Lett. 34, pp. 481-482, 1998. 